\title{
Inbred decorated crickets exhibit higher measures of macroparasitic immunity than outbred individuals
}

\author{
SN Gershman ${ }^{1}$, CA Barnett ${ }^{1}$, AM Pettinger ${ }^{1}$, CB Weddle ${ }^{1}$, J Hunt ${ }^{2}$ and SK Sakaluk ${ }^{1}$ \\ ${ }^{1}$ Behavior, Ecology, Evolution and Systematics Section, School of Biological Sciences, Illinois State University, Normal, IL, USA and \\ ${ }^{2}$ Centre for Ecology and Conservation, School of Biosciences, University of Exeter, Cornwall Campus, Penryn TR10 9EZ, Cornwall, UK
}

\begin{abstract}
Inbreeding is assumed to have negative effects on fitness, including the reduced ability to withstand immune challenges. We examined the immunological consequences of inbreeding in decorated crickets, Gryllodes sigillatus, by comparing lytic activity, phenoloxidase (PO) activity, and encapsulation ability of crickets from eight inbred lines with that of crickets from the outbred founder population. Surprisingly, crickets from inbred lines had a greater encapsulation ability compared with crickets from the outbred population. We suggest that because inbred crickets have reduced reproductive effort, they may, therefore, have the option of devoting more resources to this form of immunity than outbred individuals. We also found that
\end{abstract}

both inbred and outbred females had higher immunity than males in PO activity and implant darkness. This result supports the hypothesis that females should devote more effort to somatic maintenance and immunity than males. PO activity and implant darkness were heritable in both males and females, but lytic activity was only heritable in females. Males and females differed in the heritability of, and genetic correlations among, immune traits, suggesting that differences in selective pressures on males and females may have resulted in a sexual conflict over optimal immune trait values.

Heredity (2010) 105, 282-289; doi:10.1038/hdy.2010.1; published online 3 February 2010

Keywords: crickets; genetic correlations; heritability; inbreeding depression; immunity; sexual conflict

\section{Introduction}

Inbreeding leads to the increased probability that deleterious alleles will be found in the homozygous condition, resulting in reduced fitness (Charlesworth and Charlesworth, 1987). Inbred populations founded by relatively few individuals often lack the genetic variation needed to respond to environmental change (Keller and Waller, 2002). Novel parasites and pathogens are particularly pervasive forms of environmental change and represent a rapidly evolving selective force on host populations. The ability to draw on a large pool of genetic variation allows hosts to respond adaptively to such challenges and thus inbreeding places populations at a competitive disadvantage by depriving them of their ability to co-evolve defenses against pathogenic invaders (Stow and Beattie, 2008). Moreover, inbreeding can have negative effects on overall health as genetic drift allows disadvantageous traits to reach fixation (Reid et al., 2003; Calleri et al., 2006; Ilmonen et al., 2008). Consequently, inbred individuals may have poorly adapted or disabled immune pathways or fewer resources available with which to respond to novel parasites and pathogens (Rantala and Roff, 2007).

Immunity is not a single trait, but represents a complex network of protective mechanisms that may or may not

Correspondence: Dr SN Gershman, Behavior, Ecology, Evolution and Systematics Section, School of Biological Sciences, Illinois State University, Julian 210, Box 4120, Normal, IL 61790-4120, USA.

E-mail: sgershm@ilstu.edu

Received 31 July 2009; revised 3 December 2009; accepted 21

December 2009; published online 3 February 2010 be related to one another (Adamo, 2004a). For example, the ability of an individual to mount an immune response may reflect their general health, such that one individual may be superior in multiple components of immunity to another individual. However, immune components often draw on the same pool of resources or exhibit negative genetic correlations, trading off against one another as do other life history traits (Cotter et al., 2004; Rantala and Roff, 2005). These trade-offs may be affected by inbreeding because inbreeding decreases the ability of individuals to use available resources, which can lead to a decrease in the maximal size and growth rates of individuals (Keller and Waller, 2002; Rantala and Roff, 2006). If a trade-off is resource based, the reduced availability of resources may reveal tradeoffs between traits that are otherwise masked in outbred individuals (Tatar and Carey, 1995).

Inbreeding can also differentially affect the immune responses of the sexes. For example, female vertebrates have been found to have higher immunity compared with males because of the immunosuppressive effects of testosterone (for example, Roberts et al., 2004). Crickets lack sex-specific hormones, but there have been several studies showing that females exhibit more robust immune responses than males (Gray, 1998; Adamo et al., 2001; Rantala and Roff, 2006; Gershman, 2008). This effect may be due to fundamental differences in life history strategies (Zuk, 1990; Nunn et al., 2009): although a male's reproductive success is determined primarily by the number of females with which he mates, a female's reproductive success depends largely on the number of eggs she can produce (Bateman, 1948). Thus, males maximize their fitness by investing in mating effort at the 
expense of longevity. Females, in contrast, achieve greater lifetime reproduction through increased longevity and should, therefore, invest more in maintenance activities such as immune function. Thus, the optimal investment in immunity for females is predicted to be higher than that of males (Zuk, 1990). However, the effect of inbreeding on sex-specific effects of immunity has received little attention. If inbreeding has the potential to depress immune responsiveness, then we might expect this effect to impact more heavily on females and that inbred populations would show decreased sex-specific differences in immunity.

In this study, we examine the effects of inbreeding on immune responses of decorated crickets, Gryllodes sigillatus, by comparing the immune responses of individuals between and within inbred and outbred populations. Specifically, we (1) determine the effect of inbreeding on different components of immune function, (2) identify possible trade-offs between these components, (3) assess the effects of inbreeding on any sexspecific differences in immunity, (4) measure genetic variation in immune function and genetic correlations among components of immunity, and (5) examine the genetic correlations between components of immunity between the sexes. We discuss our findings with regard to the implications of inbreeding for immunity and the evolution of immune function in the sexes.

\section{Materials and methods}

\section{Creation of lines}

G. sigillatus used in this study were the descendents of $\sim 500$ adult crickets collected in Las Cruces, New Mexico in 2001, and used to initiate a laboratory colony maintained at a population size of $\sim 5000$ and allowed to breed panmictically (hereafter, the 'outbred' population). Nine inbred lines (designated A-I), eight of which (A, B, D-I) were used in this study, were created by subjecting crickets, randomly selected from the large, panmictic population described above, to 23 generations of full-sib mating followed by 3-4 generations of panmixis within each line (Ivy et al., 2005). Crickets were held in 55-L plastic storage bins in an environmental chamber maintained at $28-30{ }^{\circ} \mathrm{C}$ on a $14: 10 \mathrm{~h}$ light/ dark cycle. Densities of crickets per box were kept constant among lines, with each box producing $\sim 130$ adults per generation. Identical densities were maintained in the outbred colony boxes. Measurements made in 2007 revealed evidence of significant inbreeding depression in the inbred lines even after 17 generations of inbreeding: inbred lines all showed lower hatching success, decreased offspring production, and longer developmental times compared with the outbred population (St John JM and Sakaluk SK, unpublished data).

Crickets were provisioned with Flukers cricket chow, water provided in $40-\mathrm{ml}$ plastic tissue culture flasks plugged with cotton dental rolls, and egg cartons to provide shelter and to increase surface area. The food for both nymphs and adults was always provided in excess because variation in the quality of pre-adult diet can have positive or negative effects on the ability of individuals to mount an immune response (Ojala et al., 2005; Klemola et al., 2007; Smilanich et al., 2009).
Moistened peat moss provided in small plastic containers was made available both as an oviposition substrate and as a source of additional water.

\section{Measurement of immune responses}

The insect immune system comprises both humoral and cell-mediated components. Humoral responses include the production of antimicrobial compounds that defend against bacterial, viral, or fungal pathogens (Beckage, 2008). Cell-mediated responses include the encapsulation or melanization of foreign objects within the body cavity, which occurs as a result of the accumulation of melanin and hemocytes. In this study, we examined both cellmediated and humoral responses. First, we estimated the amount of the inactive hemolymph-bound enzyme, phenoloxidase (PO). PO catalyzes the reaction of dopamine into melanin, a key step in the encapsulation response pathway (Söderhäll and Cerenius, 1998). Second, we estimated the amount of a hemolymphbound lysozyme-like enzyme, which defends against bacteria. Third, we measured the degree to which a foreign body was engulfed by hemocytes and melanized, providing a measure of macroparasitic defense. Encapsulation ability was measured in vivo as the response of individuals to a novel immune challenge, and thus represents a 'realized' immune response. However, estimates of lysozyme and PO are 'potential' responses because they measure in vitro the amount of enzyme present, providing information about the potential of an individual to mount an immune response. PO activity (Adamo et al., 2001; Siva-Jothy et al., 2001; Fedorka and Zuk, 2005; Leman et al., 2009), lytic activity (Rantala and Kortet, 2003; Cotter et al., 2004; Gershman, 2008), and implant encapsulation (Siva-Jothy et al., 2001; Rantala and Kortet, 2003; Zuk et al., 2004; Leman et al., 2009) are commonly used to assess insect immunity.

Data for lytic activity, PO activity, and implant darkness were obtained from between 52 and 67 individuals from each of the inbred lines, and from 67 individuals from the outbred population. This represents a total of 249 males and 240 females. Crickets were removed from the outbred population or inbred lines within $48 \mathrm{~h}$ of adult eclosion. To ensure that crickets were sexually experienced, males and females of the same age cohort were housed together for 6 days. Eight to 9 days after adult eclosion, crickets were weighed and housed individually. Twenty-four hours later, crickets were implanted with a 3-mm long segment of $0.255-\mathrm{mm}$ diameter nylon monofilament fishing line that had been abraded with sandpaper. A small hole was made ventrally between the fifth and sixth abdominal segments with a 27-gauge syringe needle, and the implant was inserted until it was completely contained within the cricket's abdominal cavity. Before implantation, crickets were cold anesthetized for $10 \mathrm{~min}$ at $6^{\circ} \mathrm{C}$ in a refrigerator, and implants and needles were sterilized in $70 \%$ ethanol. These procedures were similar to those used in other studies (Siva-Jothy et al., 1998; Rantala et al., 2000; Doums et al., 2002; Fedorka et al., 2004; Zuk et al., 2004). After implantation, crickets were returned to their individual containers in the environmental chamber and allowed to resume normal activity. A pilot study of 10 crickets demonstrated that $48 \mathrm{~h}$ was the most appropriate duration for the implants to remain in the crickets to 
provide a range of variation in melanization. Exactly $48 \mathrm{~h}$ after each cricket was implanted, hemolymph samples were drawn, after which crickets were frozen and stored in a $-80^{\circ} \mathrm{C}$ freezer; $3 \mu \mathrm{l}$ of hemolymph were mixed with $40 \mu \mathrm{l}$ of phosphate-buffered saline solution and frozen in the $-80^{\circ} \mathrm{C}$ freezer to induce cell lysis and to prevent enzymatic reactions from proceeding. Hemolymph samples were stored at $-80^{\circ} \mathrm{C}$ for at least 1 week before analysis.

Implants were dissected out of the frozen crickets, and any clumps of tissue removed. Each implant was photographed three times from three different sides next to a clean implant control using a Nikon Coolpix 4500 digital camera (Nikon Inc., Melville, NY, USA) mounted on a Wild Heerbrugg dissecting microscope. Each implant and control was outlined using the ImageJ (http://rsbweb.nih.gov/ij/) polygon tool. The darkness of each implant and control was measured as the average grayscale value of all of the pixels within each image using the same software. The darkness score for each individual was calculated as the average difference of the three implants darkness scores subtracted from the controls. Thus, darker implants yielded higher darkness scores.

To measure PO activity of hemolymph samples drawn from experimental individuals, a known quantity of L-DOPA was added to hemolymph to replace the naturally occurring substrate. As the amount of L-DOPA is not a limiting factor in the experimental reaction leading to melanization and was constant across samples, the subsequent melanization of the hemolymph must be due to variation in the amount of $\mathrm{PO}$ present in the hemolymph of individuals. In preliminary studies of $G$. sigillatus, males had undetectably low levels of standing PO. Therefore, we activated the pro-PO for all crickets using the enzyme $\alpha$-chymotrypsin to estimate the total PO activity for each cricket (Adamo, 2004b). We combined $5 \mu$ l of hemolymph solution with $7 \mu$ l of bovine pancreas $\alpha$-chymotrypsin $\left(1.3 \mathrm{mg} \mathrm{ml}^{-1}\right.$, Sigma-Aldrich Inc., Steinheim, Germany, C7762) in each well of a spectrophotometer microplate and incubated the mixture for $20 \mathrm{~min}$ at room temperature $\left(20^{\circ} \mathrm{C}\right)$. We then added $90 \mu \mathrm{l}$ of $15 \mathrm{mM}$ L-DOPA (Sigma-Aldrich Inc., D9628-5G), and recorded optical density (OD) at $490 \mathrm{~nm}$ using a PowerWave 340 Microplate Spectrophotometer with Kc4 data analysis software (Bio-Tek Instruments, Richmond, VA, USA). This method estimates the total change in OD over the course of the reaction, ranging from an OD of 0 (transparent) to 4 (opaque). OD readings were taken every $10 \mathrm{~min}$ for $210 \mathrm{~min}$. Preliminary tests indicated that readings taken between 30 and 180 min best describe the fastest rate of change in OD over time. The PO activity rate was calculated as the change in OD over time (OD/time). These protocols were adapted from Adamo (2004b), Fedorka and Zuk (2005), Shoemaker et al. (2006), and Bailey and Zuk (2008).

To estimate lytic activity, $3 \mathrm{mg}$ of Micrococcus lysodeikticus (Sigma-Aldrich Inc., M3770-5G) per $10 \mathrm{ml}$ of phosphate-buffered saline buffer was added to hemolymph to determine the ability of a given lysozyme to lyze the bacterial cells. Preliminary tests showed that this amount of Micrococcus caused visible cell lysis and activity to level off after $3 \mathrm{~h}$. To quantify lytic activity, $10 \mu \mathrm{l}$ of the thawed frozen hemolymph sample, along with $90 \mu \mathrm{l}$ of phosphate-buffered saline buffer containing
M. lysodeikticus, was added to each spectrophotometer microplate well and changes in OD were recorded. This method estimates the total change in OD from opaque to clear as lysozymes or lysozyme-like enzymes lyze the bacterial cells. OD readings were collected every $5 \mathrm{~min}$ for $180 \mathrm{~min}$. Preliminary tests indicated that readings between 30 and $120 \mathrm{~min}$ produced a measurable and consistent change in OD/time. Although OD decreases as more bacterial cells are lyzed, lytic activity is given as a positive number for clarity. This experimental design does not permit the characterization of the specific lysozyme responsible for cell lysis (Schneider, 1985); the observed lytic activity is thus attributed to a lysozyme-like enzyme.

Eight to 12 control samples lacking insect hemolymph were included in the spectrophotometric analysis of each 96-sample plate to detect any non-experimental change in OD/time across plates. Control samples did not differ significantly among sample plates for lytic activity (ANOVA $\mathrm{F}_{6,72}=1.56, P=0.18$ ). There was, however, an effect of plate on PO OD over time (ANOVA $\mathrm{F}_{7,82}=43.1$, $P<0.0001)$. To control for differences among plates, lytic activity and $\mathrm{PO} \mathrm{OD} /$ time were subtracted from the average control OD/time of the plate. Two PO activity scores were removed from the analysis as extreme outliers. Without these two data points, all dependent variables were normally distributed and homoscedastic.

\section{Statistical analyses}

MANCOVA was used to assess the effects of sex and body mass on components of immunity in outbred crickets, and to determine the effects of sex, mass, and line on components of immunity in inbred crickets. Interaction effects were included in the initial models, but were omitted from the final models because they were not statistically significant. For comparisons between inbred and outbred crickets, it was necessary to correct for the possibility that results could have been confounded by levels of genetic relatedness between individuals within lines. Individuals within inbred lines share a single genetic origin and so cannot be considered genetically independent. Outbred populations are likely to be considerably more genetically heterogeneous compared with inbred lines. Thus, immune responses of inbred and outbred crickets were compared by performing a multivariate contrast between the multivariate average of components of immunity for each line versus the components of immunity for outbred individuals.

To examine the relationships between different components of immunity, we calculated pairwise Pearson correlations between lytic activity, PO activity, and implant darkness, using the sequential Bonferroni correction for multiple tests. The heritability of each immune response was determined as the coefficient of intraclass correlation as calculated from an ANOVA of inbred lines following the protocol outlined in David et al. (2005). Genetic correlations and their standard errors were estimated using the jackknife method of Roff and Preziosi (1994). In short, this procedure first estimates the genetic correlation between two traits using inbred line means. A sequence of $N$ (in our case 9) pseudo-values is then computed by dropping, in turn, 
each of the lines, estimating the resulting correlations and using the formula:

$$
S_{N, i}=N r_{N}-(N-1) r_{N-1, i},
$$

where $S_{N, i}$ is the $i$ th pseudo-value, $r_{N}$ is the genetic correlation estimated using the means of all $N$ inbred lines, and $r_{N-1, i}$ is the genetic correlation obtained by dropping the $i$ th inbred line alone (Roff and Preziosi, 1994). The jacknife estimate of the genetic correlation $\left(r_{j}\right)$ is then simply the mean of the pseudo-values, and an estimate of the standard error (s.e.) is given by

$$
\text { s.e. }=\frac{\sum_{i=1}^{i=N}\left(S_{N, i}-r_{j}\right)^{2}}{N(N-1)}
$$

Using simulation models, Roff and Preziosi (1994) showed that this jacknife approach provides better genetic estimates than those based on conventional inbred line means when the number of inbred lines contained in the analysis is small ( $<20$ lines). It is important to note that estimates of genetic (co)variance from inbred lines contain variance because of dominance and/or epistasis and therefore should be considered broad-sense estimates (Falconer and Mackay, 1996). Moreover, because crickets from each inbred line were reared in the same container, it is possible that some of the observed variance between inbred lines was due to common environmental effects. However, we believe that such common environmental effects were minimal in our experiment because each inbred line was maintained under identical conditions within the same growth chamber. Heritabilities and genetic correlations were considered statistically significant if values divided by s.e. were greater than 1.96 , rejecting the null hypothesis of no correlation with a two tailed $t$-distribution and infinite degrees of freedom. We used a Mantel's test, with 10000 permutations, to determine whether our estimates of heritability and genetic correlations for immune components and body mass differed significantly between the sexes (Manly, 1997).

\section{Results}

\section{Outbred population}

PO activity and implant darkness were positively correlated in females $(r=0.48, n=32, P=0.0056)$, but not in males $(r=0.09, n=29, P=0.63)$. There was no significant correlation between lytic activity and PO activity, or lytic activity and implant darkness, in either males or females (all $P>0.05$ ).

Outbred females had higher PO activity and darker implants than males, but lytic activity did not differ between the sexes (Table 1; Figures 1-3). Body mass did not have a significant effect on immunity and was dropped from the final model (MANCOVA Wilks' $\left.\lambda_{3,57}=1.3, P=0.29\right)$.

\section{Inbred lines}

Body mass, sex, and line all had significant effects on immunity in inbred crickets (Table 2; Figures 1-3). Crickets of larger body mass exhibited higher PO activity, but mass had no significant effect on lytic activity or implant darkness (Table 2). Females had
Table 1 Multivariate analysis of variance of the effect of sex on immune responses of outbred decorated crickets (lytic activity, PO activity, and implant darkness), and follow-up ANOVAs on the effect of sex on each component of immunity

\begin{tabular}{lccc}
\hline & DF & F & P \\
\hline MANOVA (Wilks' $\lambda$ ) & $\mathbf{3 , 5 8}$ & $\mathbf{1 1 . 7}$ & $<\mathbf{0 . 0 0 0 1}$ \\
ANOVAs & & & \\
Lytic activity & 1,62 & 0.33 & 0.57 \\
PO activity & $\mathbf{1 , 6 2}$ & $\mathbf{2 4 . 5}$ & $<\mathbf{0 . 0 0 0 1}$ \\
Implant darkness & $\mathbf{1 , 6 2}$ & $\mathbf{2 3 . 4}$ & $<\mathbf{0 . 0 0 0 1}$ \\
\hline
\end{tabular}

Abbreviation: PO, phenoloxidase.

Significant effects are shown in bold.

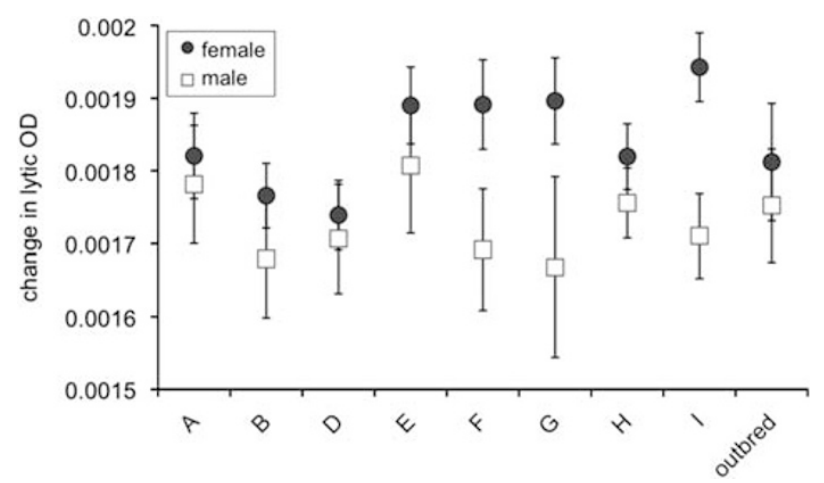

Figure 1 The effect of sex and line on lytic activity. Mean \pm s.e. of lytic activity of females (dark circles) and males (white squares) from inbred lines (A, B, D-I) and the outbred population.

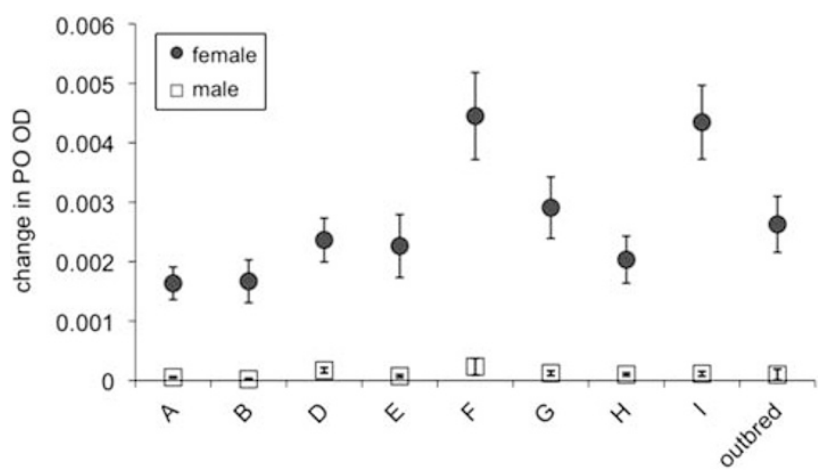

Figure 2 The effect of sex and line on phenoloxidase (PO) activity. Mean \pm s.e. of $\mathrm{PO}$ activity of females (dark circles) and males (white squares) from inbred lines (A, B, D-I) and the outbred population.

higher PO activity and implant darkness, and marginally higher lytic activity than males (Table 2; Figures 1-3). Inbred lines showed significant differences in PO activity and implant darkness, but not lytic activity (Table 2; Figures 1-3).

There were no significant correlations between the three components of immunity in males or females within inbred lines, nor were there any significant correlations when pooling across lines (all $P>0.05$ ).

\section{Inbred versus outbred crickets}

Inbred crickets had darker implants than outbred crickets, but inbred and outbred crickets did not differ 


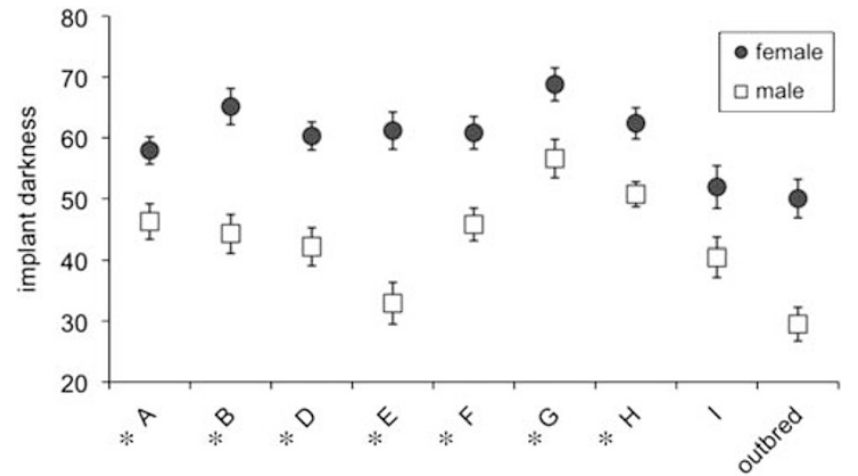

Figure 3 The effect of sex and line on implant darkness. Mean \pm s.e. of implant darkness of females (dark circles) and males (white squares) from inbred lines (A, B, D-I) and the outbred population. Asterisks indicate lines that are significantly different from the outbred colony at a level of $a<0.05$ with a sequential Bonferroni correction.

Table 2 Multivariate analysis of variance of the effects of mass, line, and sex, respectively, on immune responses of inbred decorated crickets (lytic activity, PO activity, and implant darkness), and follow-up ANOVAs on the effect of each of these factors on each component of immunity

\begin{tabular}{lccc}
\hline Source & DF & $\mathrm{F}$ & $\mathrm{P}$ \\
\hline Mass & & & \\
MANOVA (Wilks' $\lambda$ ) & $\mathbf{3 , 4 1 2}$ & $\mathbf{6 . 0 2}$ & $<\mathbf{0 . 0 0 0 1}$ \\
ANOVAs & & & \\
Lytic activity & 1,424 & 1.05 & 0.31 \\
PO activity & $\mathbf{1 , 4 2 4}$ & $\mathbf{1 6 . 3}$ & $<\mathbf{0 . 0 0 0 1}$ \\
Implant darkness & 1,424 & 1.01 & 0.32 \\
& & & \\
Line & & & \\
MANOVA (Wilks' $\lambda$ ) & $\mathbf{3 , 1 1 8 4}$ & 3.34 & $<\mathbf{0 . 0 0 0 1}$ \\
ANOVAs & & & \\
Lytic activity & 1,424 & 0.89 & 0.51 \\
PO activity & $\mathbf{1 , 4 2 4}$ & $\mathbf{4 . 7 0}$ & $<\mathbf{0 . 0 0 0 1}$ \\
Implant darkness & $\mathbf{1 , 4 2 4}$ & $\mathbf{4 . 8 9}$ & $<\mathbf{0 . 0 0 0 1}$ \\
& & & \\
Sex & & & \\
MANOVA (Wilks' $\lambda$ ) & $\mathbf{3 , 4 1 2}$ & $\mathbf{4 2 . 8}$ & $<\mathbf{0 . 0 0 0 1}$ \\
ANOVAs & & & \\
Lytic activity & 1,424 & 3.86 & 0.050 \\
PO activity & $\mathbf{1 , 4 2 4}$ & $\mathbf{7 0 . 0}$ & $<\mathbf{0 . 0 0 0 1}$ \\
Implant darkness & $\mathbf{1 , 4 2 4}$ & $\mathbf{5 7 . 0}$ & $<\mathbf{0 . 0 0 0 1}$ \\
\hline
\end{tabular}

Abbreviation: PO, phenoloxidase.

Significant effects are shown in bold.

significantly in lytic and PO activity (Table 3; Figures 1-3). Univariate contrasts comparing implant darkness in each inbred line to the outbred colony revealed that seven of the eight inbred lines had significantly darker implants than the outbred colony, with a sequential Bonferroni correction for multiple comparisons at a level of $\alpha=0.05$ (Figure 3).

\section{Genetic architecture of immune parameters}

Heritability of immune parameters was assessed separately in females and males because of the observed sexspecific differences in immunity. PO activity, implant darkness, and body mass were highly heritable for both sexes, whereas lytic activity was only significantly heritable in females (Table 4). Male lytic activity had a
Table 3 Multivariate analysis of variance of the effect of inbreeding (multivariate contrast between means of inbred lines versus outbred individuals) on immune responses of decorated crickets (lytic activity, PO activity, and implant darkness)

\begin{tabular}{lccc}
\hline & DF & F & P \\
\hline MANOVA (Wilks' $\lambda$ ) & $\mathbf{3 , 4 7 5}$ & $\mathbf{1 0 . 3}$ & $<\mathbf{0 . 0 0 0 1}$ \\
ANOVAs & & & \\
Lytic activity & 1,477 & 0.02 & 0.88 \\
PO activity & 1,477 & 0.06 & 0.80 \\
Implant darkness & $\mathbf{1 , 4 7 7}$ & $\mathbf{2 6 . 7}$ & $<\mathbf{0 . 0 0 0 1}$ \\
\hline
\end{tabular}

Abbreviation: $\mathrm{PO}$, phenoloxidase.

Follow-up ANOVAs assess the effect of inbreeding on each component of immunity. Significant effects are shown in bold.

Table 4 Heritability ( \pm s.e.) of components of immunity and body mass, and genetic correlations between traits

\begin{tabular}{lcc}
\hline \multicolumn{1}{c}{ Trait } & Female & Male \\
\hline Heritabilities & & \\
Lytic activity & $\mathbf{0 . 4 4} \pm \mathbf{0 . 1 4}$ & - \\
PO activity & $\mathbf{0 . 8 0} \pm \mathbf{0 . 0 9}$ & $\mathbf{0 . 5 1} \pm \mathbf{0 . 1 4}$ \\
Implant darkness & $\mathbf{0 . 6 5} \pm \mathbf{0 . 1 3}$ & $\mathbf{0 . 8 1} \pm \mathbf{0 . 0 9}$ \\
Body mass & & \\
& & \\
Genetic correlations & $\mathbf{0 . 7 1} \pm \mathbf{0 . 0 3}$ & - \\
Lytic activity $\times$ PO & $-0.37 \pm 0.27$ & - \\
Lytic activity $\times$ implant darkness & $\mathbf{0 . 2 3} \pm \mathbf{0 . 0 8}$ & - \\
Lytic activity $\times$ body mass & $-\mathbf{0 . 4 9} \pm \mathbf{0 . 1 9}$ & $\mathbf{0 . 1 5} \pm \mathbf{0 . 0 6}$ \\
PO $\times$ implant darkness & $\mathbf{0 . 2 1} \pm \mathbf{0 . 0 9}$ & $\mathbf{0 . 3 6} \pm \mathbf{0 . 0 6}$ \\
PO $\times$ body mass & $\mathbf{0 . 5 5} \pm \mathbf{0 . 1 1}$ & $\mathbf{0 . 6 0} \pm \mathbf{0 . 1 3}$ \\
Implant darkness $\times$ body mass & & \\
& & \\
Genetic correlations between the sexes & $\mathbf{0 . 3 4} \pm \mathbf{0 . 0 8}$ & \\
PO & $\mathbf{0 . 5 7} \pm \mathbf{0 . 1 1}$ & \\
Implant darkness & $\mathbf{0 . 9 5} \pm \mathbf{0 . 0 0 5}$ & \\
Body mass & & \\
\hline Abberiatio &
\end{tabular}

Abbreviation: PO, phenoloxidase.

Male lytic activity had a negative heritability, which was rounded to 0 and not used in estimates of genetic correlations. Significant values are shown in bold.

negative heritability, which was set to 0 and excluded from estimates of genetic correlations. Genetic correlations were also assessed separately for males and females. Females exhibited strong genetic correlations among all components of immunity, with PO activity and lytic activity being strongly positively correlated, whereas PO activity was negatively correlated with implant darkness (Table 4). All genetic correlations between components of immunity, and between immune measures and body mass, were positive in males (Table 4). A Mantel's test revealed that the estimates of heritability and genetic correlations between immune components and body mass differed significantly between males and females (vector correlation $=0.59$, $P=0.031$ ). Furthermore, all genetic correlations of immune components and body mass between the sexes were positive and significant, but each was significantly lower than one (Table 4).

\section{Discussion}

Although inbreeding typically results in reduced fitness (Charlesworth and Charlesworth, 1987; Keller and 
Waller, 2002), our results suggest that inbreeding had a positive effect on one component of immunity (encapsulation ability) in the decorated cricket, G. sigillatus. Individuals from inbred lines showed, on average, a greater ability to encapsulate foreign bodies than outbred individuals suggesting an improved ability to subdue an invading macroparasite. However, inbred and outbred crickets did not differ in either PO or lytic activity suggesting that the positive effects of inbreeding on immune function in this species are limited to encapsulation ability. This is not altogether surprising given that there is unlikely to be a single measure of immune function that provides a reliable estimate of overall immunity, as the different components of immunity frequently trade against each other, as well as with other important life history traits (Sheldon and Verhulst, 1996). Nevertheless, our findings do suggest that caution should always be taken when making the assumption that inbreeding will reduce immune function and other important fitness-related traits.

The observed relationship between inbreeding and encapsulation activity could be explained if G. sigillatus trade off investment of resources in reproduction against investment in this form of immunity. In many instances, an experimental reduction in reproduction leads to increased investment in life history traits related to somatic maintenance and survival. For example, experimental limitation of diet resulted in reduced reproduction in Drosophila melanogaster, but increased longevity (Chippindale et al., 1993). Conversely, an experimental increase in diet quality in field crickets, Teleogryllus commodus, resulted in reduced male longevity but increased reproductive effort (Hunt et al., 2004). Similar trade-offs have been found in other taxa: when reproduction is experimentally limited, survival often increases (Partridge et al., 1987; Tatar et al., 1993; Ellers et al., 2000; Hosken, 2001; McKean and Nunney, 2001). Although our study does not provide direct evidence of a trade-off between fecundity and immunity, previous measurements on the same inbred lines and outbred population of $G$. sigillatus have demonstrated that inbred individuals have lower reproductive investment than outbred colony individuals (St John JM and Sakaluk SK, unpublished data). If G. sigillatus experience a trade-off between immunity and reproduction, inbreeding, which leads to a decrease in fecundity and other components of reproductive effort, may allow these individuals to allocate more effort to immunity or other aspects of somatic maintenance. Furthermore, inbred individuals tend to mate at a lower rate than outbred individuals (Gershman SN, Weddle CB, and Sakaluk SK, unpublished data). If mating either trades off with, or is harmful to, somatic maintenance (Rolff and Siva-Jothy, 2002), inbred individuals may have lower reproductive costs and have additional resources available to devote to immunity. In the experimental design used in this study, individuals were allowed to mate ad libitum and females were allowed unlimited access to oviposition substrate. Thus, the immunological results of this study reflect both physiological and behavioral differences among inbred and outbred individuals.

Alternatively, studies on gene expression and metabolite production associated with immunity in D. melanogaster suggest that inbreeding can act similarly to an environmental stressor, activating some components of immunity (Kristensen et al., 2005; Pedersen et al., 2008). Thus, the increased encapsulation ability of inbred individuals in this study may reflect a generalized stress response to inbreeding. It is possible that sampling error inherent to any founding event could explain why inbred individuals had better immunity than outbred individuals. The inbred lines were each initiated by a pair of crickets taken from the outbred colony, and therefore it is possible (although statistically unlikely) that the 16 individuals randomly chosen from the colony had higher immunity relative to the colony mean. It is also possible that the outbred colony, despite having been maintained under constant conditions with a large breeding population (several thousand individuals), has evolved reduced immunity. If this were the case, our inbred lines may represent the average immunity of the founding population, with our outbred stock population having evolved an average immunity below this value.

In both inbred and outbred crickets, females were found to have increased immunity to males in all measures in which there were differences among the sexes. This provides support for the hypothesis that females should invest more in somatic maintenance and immunity than males (Zuk, 1990; Rolff, 2002; Nunn et al., 2009). If there is a trade-off between reproduction and longevity, males benefit from increased investment in immediate reproduction at the expense of future longevity. Females, however, benefit more than males from investing in somatic maintenance and immunity because females must survive long enough to provision and oviposit eggs (Trivers, 1972; Andersson, 1994). There is an additional reason to expect that male decorated crickets might be required to devote a large proportion of their resources to reproduction: the spermatophore transferred by male G. sigillatus at mating includes a large gelatinous mass, the spermatophylax, which the female detaches and consumes after mating (Sakaluk, 1984). Although the female feeds on this nuptial gift, sperm are evacuated into her reproductive tract from the remaining portion of the spermatophore, the sperm ampulla. Smaller spermatophylaxes require less time to consume, and males providing such gifts are penalized in the form of premature ampulla removal and reduced sperm transfer (Sakaluk, 1984, 1985, 1987), resulting in lower fertilization success (Sakaluk, 1986; Sakaluk and Eggert, 1996). Thus, male G. sigillatus should be under strong selective pressure to maximize reproductive effort at the expense of other life history traits (Gershman et al., in press).

Body mass had a significant effect on immunity in both sexes, with larger individuals exhibiting higher immunity than smaller ones (see also Rantala et al., 2004). Although females had greater body mass than males, there was no statistically significant interaction between sex and body mass in their effects on immunity. It may be that individuals who have a superior ability to acquire or use resources are able to attain both a heavier body mass as well as mount a superior immune response (van Noordwijk and de Jong, 1986). Alternatively, attaining a larger body size may provide individuals with a greater volume of hemolymph and melanin-producing enzymes. This conjecture is supported by the fact that heavier males had darker implants but not higher PO activity: in 
the encapsulation assay, implants were identical in size, but crickets varied in body size potentially giving an advantage to large individuals. The assay used in measuring PO activity, however, required an identical volume of hemolymph per individual.

Analysis of inbred lines revealed sexual differences in the heritability of, and genetic correlations among, components of immunity. PO activity and implant darkness (assays indicating the ability to combat macroparasites and parasitoids) were highly heritable for both males and females. However, implant darkness was more strongly heritable than PO activity in males, whereas the reverse was true for females. Further, although females exhibited a strong negative genetic correlation between PO activity and implant darkness, males showed a weak positive correlation between these traits. This pattern suggests sexual conflict over the optimal level of resources allocated to male and female immune components. The relatively weak genetic correlations between immune components for the sexes compared with the strong correlation between male and female body mass are also indicative of a conflict between the sexes over immunity. Moreover, because each of these genetic correlations was significantly less than one, it suggests that components of immunity have the potential to evolve independently in the sexes to attain their sex-specific optima (Via and Lande, 1985).

The most striking sexual difference in genetics underlying immunity was in the heritability of lytic activity (assaying the ability to combat bacterial infection). Although lytic activity was significantly heritable in females, it was not in males (see Rantala and Roff, 2006 for another example of extremely low heritability of lytic activity in male crickets), suggesting that males and females are subject to different selective pressures. If males experience a trade-off between resources devoted to lytic activity and reproduction, it may benefit males of all genotypes to devote less resources to lytic activity, resulting in the phenotypic expression of lytic activity that varies as much within as among our genetic lines. A companion study reveals that male G. sigillatus trade off lytic activity against spermatophylax mass (Gershman et al., in press), potentially supporting this hypothesis. Alternatively, if much stronger selection acts on this component of immunity in males than in females, this would erode any standing genetic variance for lytic activity in males but not in females.

In conclusion, we show that inbred crickets have greater encapsulation ability than outbred crickets in G. sigillatus. Our findings suggest, therefore, that the assumption that inbreeding depresses all aspects of physiology is overly simplistic and fails to address all possible trade-offs that may exist between life history traits. Although our previous work suggests that inbred individuals have lower fitness than outbred individuals in this species, life history trade-offs may allow inbred individuals to outperform their outbred conspecifics in some vital physiological traits. Furthermore, we documented clear differences between the sexes in not only phenotypic measures of immunity, but also in genotypic measures. To understand the causes of these differences, future studies are needed that focus on interactions between immunity and other sex-specific life history traits such as female fecundity and male mating effort.

\section{Conflict of interest}

The authors declare no conflict of interest.

\section{Acknowledgements}

We thank Steve Juliano for statistical advice, Tracie Ivy for her initial work in establishing the inbred lines used in this project, and Laura Vogel for her technical advice. This research was funded by grants from the National Science Foundation to SKS and CG Hamaker (IOS0543254 and IOS-0718140), and a Royal Society University Fellowship to JH. AMP was supported by a graduate fellowship from the Program of Excellence in Biomathematics at Illinois State University. SNG and CAB were supported by postdoctoral fellowships from the Program of Excellence in Neuroscience and Behavior at Illinois State University.

\section{References}

Adamo SA (2004a). How should behavioural ecologists interpret measurements of immunity? Anim Behav 68: 1443-1449.

Adamo SA (2004b). Estimating disease resistance in insects: phenoloxidase and lysozyme-like activity and disease resistance in the cricket Gryllus texensis. J Insect Physiol 50: 209-216.

Adamo SA, Jensen M, Younger M (2001). Changes in lifetime immunocompetence in male and female Gryllus texensis (formerly G. integer): trade-offs between immunity and reproduction. Anim Behav 62: 417-425.

Andersson MB (1994). Sexual Selection. Princeton University Press: Princeton, NJ.

Bailey NW, Zuk M (2008). Changes in immune effort of male field crickets infested with mobile parasitoid larvae. J Insect Physiol 54: 96-104.

Bateman AJ (1948). Intra-sexual selection in Drosophila. Heredity 2: 349-368.

Beckage NE (2008). Insect Immunity. Elsevier Inc., Academic Press: San Diego, CA.

Calleri DV, Reid EM, Rosengaus RB, Vargo EL, Traniello JFA (2006). Inbreeding and disease resistance in a social insect: effects of heterozygosity on immunocompetence in the termite Zootermopsis angusticollis. Proc R Soc Lond B 273: 2633-2640.

Charlesworth D, Charlesworth B (1987). Inbreeding depression and its evolutionary consequences. Annu Rev Ecol Syst 18: 237-268.

Chippindale AK, Leroi AM, Kim SB, Rose MR (1993). Phenotypic plasticity and selection in Drosophila life-history evolution. 1. Nutrition and the cost of reproduction. J Evol Biol 6: 171-193.

Cotter SC, Kruuk LEB, Wilson K (2004). Costs of resistance: genetic correlations and potential trade-offs in an insect immune system. J Evol Biol 17: 421-429.

David JR, Gibert P, Legout H, Petavy G, Capy P, Moreteau B (2005). Isofemale lines in Drosophila: an empirical approach to quantitative trait analysis in natural populations. Heredity 94: 3-12.

Doums C, Moret Y, Benelli E, Schmid-Hempel P (2002). Senescence of immune defense in Bombus workers. Ecol Entomol 27: 138-144.

Ellers J, Driessen G, Sevenster JG (2000). The shape of the tradeoff function between egg production and life span in the parasitoid Asobara tabida. Neth J Zool 50: 29-36.

Falconer DS, Mackay TFC (1996). Introduction to Quantitative Genetics, 4th edn. Prentice Hall: England.

Fedorka KM, Zuk M, Mousseau TA (2004). Immune suppression and the cost of reproduction in the ground cricket, Allonemobius socius. Evolution 58: 2478-2485.

Fedorka KM, Zuk M (2005). Sexual conflict and female immune suppression in the cricket, Allonemobious socius. J Evol Biol 18: $1515-1522$ 
Gershman SN (2008). Sex-specific differences in immunological costs of multiple mating in Gryllus vocalis field crickets. Behav Ecol 19: 810-815.

Gershman SN, Barnett CA, Pettinger AM, Weddle CB, Hunt J, Sakaluk SK (in press). Give 'til it hurts: trade-offs between immunity and male reproductive effort in the decorated cricket, Gryllodes sigillatus. J Evol Biol.

Gray DA (1998). Sex differences in susceptibility of house crickets, Acheta domesticus, to experimental infection with Serratia liquefaciens. J Invertebr Pathol 71: 288-289.

Hosken DJ (2001). Sex and death: microevolutionary trade-offs between reproductive and immune investment in dung flies. Curr Biol 11: R379-R380.

Hunt J, Brooks R, Jennions MD, Smith MJ, Bentsen CL, Bussiere LF (2004). High-quality male field crickets invest heavily in sexual display but die young. Nature 432: 1024-1027.

Ilmonen P, Penn DJ, Damjanovich K, Clarke J, Lamborn D, Morrison L (2008). Experimental infection magnifies inbreeding depression in house mice. J Evol Biol 21: 834-841.

Ivy TM, Weddle CB, Sakaluk SK (2005). Females use selfreferent cues to avoid mating with previous mates. Proc $R$ Soc Lond B 272: 2475-2478.

Keller LF, Waller DM (2002). Inbreeding effects in wild populations. Trends Ecol Evol 17: 230-241.

Klemola N, Klemola T, Rantala MJ, Ruuhola T (2007). Natural host-plant quality affects immune defense of an insect herbivore. Entomol Exp Appl 123: 167-176.

Kristensen NK, Sørensen P, Kruhøoffer M, Pedersen KS, Loeschcke V (2005). Genome-wide analysis on inbreeding effects on gene expression in Drosophila melanogaster. Genetics 171: 157-167.

Leman JC, Weddle CB, Gershman SN, Kerr AM, Ower GD, St John JM et al. (2009). Lovesick: immunological costs of mating to male sagebrush crickets. J Evol Biol 22: 163-171.

Manly BFJ (1997). Randomization, Bootstrap and Monte Carlo Methods in Biology, 2nd edn. Chapman \& Hall, CRC Press: Boca Raton, FL.

McKean KA, Nunney L (2001). Increased sexual activity reduces male immune function in Drosophila melanogaster. Proc Natl Acad Sci USA 98: 7904-7909.

Nunn CL, Lindenfors P, Pursall ER, Rolff J (2009). On sexual dimorphism in immune function. Philos Trans $R$ Soc B 364: 61-69.

Ojala K, Julkunen-Tiito R, Lindstrom L, Mappes J (2005). Diet affects the immune defence and life-history traits of an Arctiid moth Parasemia plantaginis. Evol Ecol Res 7: 1153-1170.

Partridge L, Green A, Fowler K (1987). Effects of eggproduction and of exposure to males on female survival in Drosophila melanogaster. J Insect Physiol 33: 745-759.

Pedersen SP, Kristensen TN, Loeschcke V, Petersen BO, Duus JØ, Nielsen NC et al. (2008). Metabolic signatures of inbreeding at benign and stressful temperatures in Drosophila melanogaster. Genetics 180: 1233-1243.

Rantala MJ, Ahtiainen JJ, Suhonen J (2004). Fluctuating asymmetry and immune function in a field cricket. Oikos 107: 479-484.

Rantala MJ, Kortet R (2003). Courtship song and immune function in the field cricket Gryllus bimaculatus. Biol J Linn Soc Lond 79: 503-510.

Rantala MJ, Koskimaki J, Taskinen J, Tynkkynen K, Suhonen J (2000). Immunocompetence, developmental stability and wingspot size in the damselfly Calopteryx splendens L. Proc $R$ Soc Lond B 267: 2453-2457.

Rantala MJ, Roff DA (2005). An analysis of trade-offs in immune function, body size and development time in the Mediterranean field cricket, Gryllus bimaculatus. Funct Ecol 19: 323-330.

Rantala MJ, Roff DA (2006). Analysis of the importance of genotypic variation, metabolic rate, morphology, sex and development time on immune function in the cricket, Gryllus firmus. J Evol Biol 19: 834-843.

Rantala MJ, Roff DA (2007). Inbreeding and extreme outbreeding cause sex differences in immune defence and life history traits in Epirrita autumnata. Heredity 98: 329-336.
Reid JM, Arcese P, Keller LF (2003). Inbreeding depresses immune response in song sparrows (Melospiza melodia): direct and inter-generational effects. Proc R Soc Lond B 270: 2151-2157.

Roberts ML, Buchanan KL, Evans MR (2004). Testing the immunocompetence handicap hypothesis: a review of the evidence. Anim Behav 68: 227-238.

Roff DA, Preziosi R (1994). The estimation of the genetic correlation: the use of the jackknife. Heredity 73: 544-548.

Rolff J (2002). Bateman's principle and immunity. Proc $R$ Soc Lond B 269: 867-872.

Rolff J, Siva-Jothy MT (2002). Copulation corrupts immunity: a mechanism for a cost of mating in insects. Proc Natl Acad Sci USA 99: 9916-9918.

Sakaluk SK (1984). Male crickets feed females to ensure complete sperm transfer. Science 223: 609-610.

Sakaluk SK (1985). Spermatophore size and its role in the reproductive behaviour of the cricket, Gryllodes supplicans (Orthoptera: Gryllidae). Can J Zool 63: 1652-1656.

Sakaluk SK (1986). Sperm competition and the evolution of nuptial feeding behavior in the cricket, Gryllodes supplicans (Walker). Evolution 40: 584-593.

Sakaluk SK (1987). Reproductive behaviour of the decorated cricket, Gryllodes supplicans (Orthoptera: Gryllidae): calling schedules, spatial distribution, and mating. Behaviour 100: 202-225.

Sakaluk SK, Eggert A-K (1996). Female control of sperm transfer and intraspecific variation in sperm precedence: antecedents to the evolution of a courtship food gift. Evolution 50: 694-703.

Schneider PM (1985). Purification and properties of 3 lysozyme from hemolymph of the cricket, Gryllus-bimaculatus (Degeer) Insect Biochem 15: 463-470.

Sheldon BC, Verhulst S (1996). Ecological immunology: costly parasite defenses and trade-offs in evolutionary ecology. Trends Ecol Evol 11: 317-321.

Shoemaker KL, Parsons NM, Adamo SA (2006). Mating enhances parasite resistance in the cricket Gryllus texensis. Anim Behav 71: 371-380.

Siva-Jothy MT, Tsubaki Y, Hooper RE (1998). Decreased immune response as a proximate cost of copulation and oviposition in a damselfly. Physiol Entomol 23: 274-277.

Siva-Jothy MT, Tsubaki Y, Hooper RE, Plaistow SJ (2001). Investment in immune function under chronic and acute immune challenge in an insect. Physiol Entomol 26: 1-5.

Smilanich AM, Dyer LA, Chambers JQ, Bowers MD (2009). Immunological cost of chemical defence and the evolution of herbivore diet breadth. Ecol Lett 12: 612-621.

Söderhäll K, Cerenius L (1998). Role of the prophenoloxidaseactivating system in invertebrate immunity. Curr Opin Immunol 10: 23-28.

Stow A, Beattie A (2008). Chemical and genetic defenses against disease in insect societies. Brain Behav Immun 22: 1009-1013.

Tatar M, Carey JR (1995). Nutrition mediates reproductive trade-offs with age-specific mortality in the beetle Callosobruchus-maculatus. Ecology 76: 2066-2073.

Tatar M, Carey JR, Vaupel JW (1993). Long-term cost of reproduction with and without accelerated senescence in Callobruchus-maculatus-analysis of age-specific mortality. Evolution 47: 1302-1312.

Trivers RL (1972). Parental investment and sexual selection. In: Campbell B (ed). Sexual Selection and the Descent of Man, 1871-1971. Heinemann: London. pp 136-179.

van Noordwijk AJ, de Jong G (1986). Acquisition and allocation of resources: their influence on variation in life history tactics. Am Nat 128: 137-142.

Via S, Lande R (1985). Genotype-environment interaction and the evolution of phenotypic plasticity. Evolution 39: 505-532.

Zuk M (1990). Reproductive strategies and disease susceptibility -an evolutionary viewpoint. Parasitol Today 6: 231-233.

Zuk M, Simmons LW, Rotenberry JT, Stoehr AM (2004). Sex differences in immunity in two species of field crickets. Can J Zool 82: 627-634. 